\title{
Structural insights into the mechanism of ubiquitination by the linear ubiquitin chain assembly complex (LUBAC)
}

\author{
Simin Rahighi ${ }^{1}$, Soichi Wakatsuki ${ }^{2}$ \\ ${ }^{1}$ Chapman University School of Pharmacy (CUSP), Harry and Diane Rinker Health \\ Science Campus, Irvine, CA, USA. ${ }^{2}$ Department of Structural Biology, Stanford \\ University School of Medicine, Stanford, CA, USA.
}

Nuclear Factor (NF)-kB proteins are DNA transcription factors that are sequestered in the cytoplasm by binding to inhibitory molecules (inhibitors of $\kappa B$ or $\mid \kappa B s$ ). Upon stimulation, a cascade of cellular events results in the phosphorylation of $1 \kappa B s$ by the $1 \kappa B$ kinase (IKK) complex. Subsequently, IKB proteins are ubiquitinated and degraded by the proteasome, leading to activation and translocation of NF-kB proteins to the nucleus. The regulatory component of IKK complex, NF-kB essential modulator (NEMO), is indispensable for the activation of NF-kB signaling pathway. NEMO is, in turn, activated through ubiquitination by the linear ubiquitin chain assembly complex (LUBAC). The mechanism of transfer of ubiquitin molecules to NEMO by LUBAC is not, yet, understood. Here, we report a ternary complex of NEMO/linear di-ubiquitin/HOIP subunit of LUBAC. In this crystal structure, NEMO interacts with linear ubiquitin chains and NZF-1 domain of HOIP, simultaneously. While two linear di-ubiquitins bind to the either sides of a NEMO dimer, HOIP NZF-1 binds to a region upstream to the ubiquitin-binding domain. Interestingly, a ubiquitin molecule from the neighboring asymmetric unit interacts with HOIP NZF-1, with its Cterminal tail positioned adjacent to the ubiquitination site on NEMO. This suggests that HOIP NZF-1 plays a role in retaining ubiquitin in close proximity of NEMO, thereby facilitating transfer of ubiquitin by the catalytic domain of HOIP. 